\section{$\underset{\substack{\text { hommes } \\ \text { \& migrations }}}{ }$}

\section{Hommes \& migrations}

Revue française de référence sur les dynamiques

migratoires

1285 | 2010

L'appel du pied

\title{
Pour le Mondial 2010. La fin de la génération "black-blanc-beur"?
}

Entretien avec Yvan Gastaut, réalisé par Marie Poinsot

Yvan Gastaut et Marie Poinsot

\section{(2) OpenEdition}

Journals

Édition électronique

URL : http://journals.openedition.org/hommesmigrations/1170

DOI : 10.4000/hommesmigrations. 1170

ISSN : 2262-3353

Éditeur

Musée national de l'histoire de l'immigration

Édition imprimée

Date de publication : 1 mai 2010

Pagination : 6-11

ISSN : 1142-852X

\section{Référence électronique}

Yvan Gastaut et Marie Poinsot, "Pour le Mondial 2010. La fin de la génération "black-blanc-beur" ?»,

Hommes \& migrations [En ligne], 1285 | 2010, mis en ligne le 29 mai 2013, consulté le 03 mai 2019.

URL : http://journals.openedition.org/hommesmigrations/1170; DOI : 10.4000/

hommesmigrations. 1170 


\section{Pour le Mondiall 2010 La fin de la génération "black-blanc-beur"?}

Entretien avec Yvan Gastaut, maître de conférences, université de Nice réalisé par Marie Poinsot

Le 24 mai dernier, depuis Tignes où les Bleus achèvent leur stage de préparation, le sélectionneur de l'équipe de France, Raymond Domenech, dévoilait la liste des 23 joueurs en partance pour l'Afrique du Sud. Les Bleus version 2010 ont-ils quelque chose en commun avec la sélection victorieuse de 1998 ? Derrière la question de la performance se pose celle du lien passionné quil, à chaque grand rendez-vous de la planète football, se renouvelle entre la société française et son équipe nationale. 


\section{H\&M : Que pensez-vous de l’annonce de la sélection de l'équipe de France pour la Coupe du monde 2010 ?}

Yvan Gastaut: On peut d'abord insister sur l'impact médiatique de cette sélection, avec la mise en scène de l'annonce au journal de 20 heures sur TF1. Malgré les critiques, cela semble revêtir une importance capitale, comme lorsque les hommes d'État annoncent une grande décision politique. Il y a une identification forte entre l'opinion française et l'équipe de France : toutes les étapes de son parcours, de la qualification aux matchs, vont être suivies par des millions de gens. Ce rituel de la sélection s'organise à présent sur quatre ans avec les éliminatoires, les aléas de la préparation et de l'annonce. On a rappelé la manière dont a été annoncée la liste des 30 joueurs sélectionnés par Aimé Jacquet en 1998 en insistant sur le fait qu'il en avait éliminé 7 par la suite, qui ont connu des carrières difficiles et des problèmes psychologiques, comme Ibrahim Ba que l'on découvre dans l'exposition.

Ce rituel bien ordonné et bien huilé conserve une part de hasard, d'intrigue et d'aléatoire. De nombreux joueurs n'étaient pas au courant de leur sélection par Raymond Domenech. Par exemple, Yann M'Vila, joueur rennais de 19 ans et d'origine congolaise, qui n'a pas été retenu par la suite dans la liste des 23.

\section{H\&M : Retrouve-t-on dans cette sélection la même configuration qu'en 1998 ?}

Y. G. : On parle beaucoup dans l'exposition de la génération de 1987 qui a connu une ascension fulgurante en 2004-2005, avec Karim Benzema et Samir Nasri, tous deux d'origine algérienne, et Hatem Ben Arfa, d'origine tunisienne. Sur ces trois joueurs, aucun n'a été sélectionné pour jouer en Afrique du Sud. Une vraie surprise, à tel point que le ministre de l'Immigration et de l'Identité nationale, Éric Besson, a signalé cette "anomalie" selon laquelle aucun joueur d'origine maghrébine ne fait partie du groupe. Premier événement, la grande vedette, Benzema, actuellement au Real Madrid, n'est pas sélectionné en raison des relations très conflictuelles qu'il entretient avec Raymond Domenech et de ses difficultés à évoluer régulièrement dans le collectif des Galactiques où les places sont chères. Être écarté de la sélection nationale, comme l'a été Robert Pirès, d'origine espagnole et portugaise, depuis plusieurs années, est vécu comme un échec par ces joueurs. Domenech préfère choisir des remplaçants, des animateurs du groupe qui vont accepter d'avoir très peu de chances de jouer.

Le second événement, c'est la non-sélection de Patrick Vieira, grande figure du football, français d'origine capverdienne, ancien capitaine des Bleus avec 107 sélections à son actif et qui, compte tenu de son âge, a vu s'envoler sa dernière 
occasion de participer à la Coupe du monde. Au total, ce sont cinq grandes figures issues de l'immigration qui ne sont donc pas sélectionnées pour 2010.

\section{H\&M : Cette sélection caractérise-t-elle la diversité de la société française?}

Y. G. : Au mépris des conversations de café du commerce reprenant l'antienne "trop de Noirs en équipe de France", l'annonce de la sélection apparaît, il faut bien le dire, comme un non-événement quant aux origines des joueurs. Les médias n'ont pas abordé le visage des Bleus sous cet angle qui semble un brin dépassé. On ne retient à juste titre que les compétences sportives, alors que la plupart sont issus de l'immigration par leurs parents ou leurs grands-parents. Se pose aussi la question des joueurs originaires de l'Outre-mer: sont-ils ou pas des représentants de la diversité ? Le débat est ouvert. Quoi qu'il en soit, le stage de préparation des Bleus à La Réunion est emblématique d'une tendance à aller chercher du soutien dans les DOM-TOM.

Sur ce point, il n'est pas inutile de faire un parallèle avec la sélection de l'équipe algérienne qui a été annoncée quelque temps auparavant. La question était de savoir s'il fallait prendre des joueurs évoluant dans le championnat local ou des binationaux évoluant à l'étranger. Une partie de l'opinion algérienne aurait préféré avoir des joueurs "purement nationaux". Mais, logiquement, la plupart des joueurs sélectionnés viennent de clubs européens, ce qui fait dire à certains que l'équipe des Fennecs est principalement composée d'“étrangers". Malgré les critiques et les atermoiements, les Français restent néanmoins profondément attachés aux Bleus. Une élimination au premier tour serait vécue comme un échec national.

\section{H\&M : Mais l'équipe de France est aussi composée de joueurs évoluant à l'étranger?}

Y. G. : Il est vrai que le Onze de France est composé de joueurs évoluant au sein des grands clubs étrangers, tout particulièrement anglais ou espagnols. Toutefois, on remarque que l'équipe de 2010 accorde une part plus importante que précédemment à des joueurs issus de clubs français. C'est peut-être une tendance de cette sélection de privilégier les joueurs restés en France dans la mesure où les clubs semblent en progression sur l'échiquier européen.

\section{H\&M : L'équipe de France résulte-t-elle d'un choix pour des joueurs ayant la double nationalité ?}

Y. G. : Certains joueurs binationaux ont été sollicités par les équipes de leur pays d'origine, mais ils ont pour la plupart fait le pari de la sélection française. Sur le plan 
du règlement, le choix de la nationalité sportive du joueur est définitif pour tout le reste de leur carrière professionnelle, ce qui suscite de nombreuses transactions auprès des jeunes pousses : on peut noter le cas de Francisco Higuain, né à Brest parce que son père argentin était un joueur du stade brestois. Il a longuement hésité après avoir été approché par Raymond Domenech, mais a choisi l'équipe argentine. La France et l'Argentine étant de niveau équivalent dans le classement international, son choix a pu être motivé par des raisons sentimentales. Pour certains joueurs qui n'ont pas accès à l'équipe de France, la double nationalité peut favoriser une carrière internationale. Par exemple, le Lillois Ludovic Obraniak, petit-fils de Polonais, défend les couleurs de la Pologne alors qu'il n'y a jamais vécu et ne parle pas polonais.

\section{H\&M : La Coupe du monde se déroule cette année en Afrique du Sud. Cette loca- lisation a-t-elle une portée symbolique?}

Y. G.: C'est la première fois qu'un pays africain accueille la compétition internationale. Cela comporte une force symbolique très grande. Tout d'abord, parce qu'elle est organisée sur le sol africain en soulignant la capacité d'accueil ainsi que la performance sportive du football sur l'ensemble du continent africain. Deuxièmement, nous devons comprendre les dynamiques à l'ceuvre en Afrique du Sud. Dans ce pays qui est sorti de l'apartheid depuis vingt ans, le sport a une force formidable. Le soutien de Nelson Mandela à la première Coupe du monde de rugby - sport associé aux Blancs - en Afrique du Sud en 1995 et aujourd'hui au football, qui 
connaît avec l'organisation de la Coupe du monde un véritable engouement, montre l'importance du sport dans ce pays. L'organisation de la Coupe du monde est certainement très dispendieuse pour l'économie sud-africaine, mais laissera sans doute des traces très positives pour le pays, comme nous l'avons vu avec les Jeux olympiques de Pékin en 2008.

\section{H\&M : Cette Coupe du monde va-t-elle compenser le manque d'éclat de la Coupe d'Afrique des Nations qui s'est déroulée début 2010 en Angola ?}

Y. G. : Pendant très longtemps la Coupe d'Afrique des Nations a été une coupe cantonnée à son continent. Avec la Coupe du monde, cette compétition bénéficiera d'un nouveau regard de la part de l'extérieur, regard que nous commençons déjà à modifier par apport à l'évolution des pays africains. La Coupe du monde sera donc une manière de valoriser le football africain dans le monde.

\section{H\&M : On ne découvre pas le football africain en 2010 ? La place des footballeurs africains dans l'histoire du football français est pourtant ancienne?}

Y. G. : Depuis le début, l'histoire du football français est effectivement caractérisée par une présence africaine très forte. Nous pouvons prendre 1932, une date importante qui marque à la fois le début du championnat professionnel français de football et la présence des Africains dans le championnat pour leurs qualités de footballeurs. Par exemple, Raoul Diagne, fils de Blaise Diagne, député des colonies, est l'un des meilleurs footballeurs de son époque. C'est le point de départ d'une histoire commune du championnat français : les clubs de métropole et ensuite l'équipe de France ont été régulièrement animés par des joueurs du continent africain. Ces joueurs venaient des colonies jusqu'à la fin des années cinquante, notamment des attaquants avec beaucoup de talent qui ont joué dans l'équipe nationale à l'époque coloniale. Ils bénéficient de leur statut de "sujets" français leur permettant de circuler librement au sein des clubs. Après la décolonisation, la présence africaine ne faiblit pas, elle reste même une présence décisive dans les clubs jusqu'à aujourd'hui, à la différence près qu'un certain nombre de ces joueurs jouent également dans leurs équipes nationales.

\section{H\&M : Comment sont-ils recrutés ? Y a-t-il un repérage de ces jeunes joueurs en Afrique, ou bien est-ce ces joueurs qui montrent d'abord de l'intérêt pour rejoindre nos équipes en France ?}

Y. G. : L'originalité concernant le recrutement des footballeurs africains jusqu'à aujourd'hui renvoie à l'idée que l'Afrique est un vivier où l'on peut venir se "servir", 
comme pour les travailleurs immigrés. Dans ce contexte, les clubs ont considéré qu'il fallait avoir des agents sur place pour découvrir des talents qui ne pouvaient pas forcément s'exprimer sportivement dans leur village d'origine. Cette situation date des années trente et n'a jamais cessé avec des formes différentes. Aujourd'hui, on ouvre des écoles de football dans le continent africain et on crée, au sein des clubs, des postes d'agent recruteur spécialisés. L'Afrique constitue donc un réservoir pour le football français.

\section{H\&M : La réception de ces joueurs africains dans les clubs est-elle toujours facile? Quels sentiments provoquent cette présence de figu- res africaines dans le football ?}

Y. G $_{n}$ : Les footballeurs venus d'Afrique suscitent un comportement ambigu de la part des supporters. Lorsqu'ils sont efficaces sur le terrain, ces derniers n'hésitent pas à les acclamer, et il n'y a pas d'expression de racisme lorsque le succès sportif est à la clef. En revanche lorsqu'il s'agit de regarder un match de football où l'adversaire est composé d'un certain nombre de footballeurs venus d'Afrique, les tribunes sont le lieu d'un racisme parfois très primaire, d'un comportement stéréotypé de violences verbales. Cela s'est manifesté dès les années trente en France, prenant une ampleur à partir des années quatre-vingt, à tel point qu'un gardien ivoirien, Joseph Antoine Bell, a mobilisé la Fédération française de football et un certain nombre de clubs qui ont commencé à prêter attention à ce problème de racisme dans les stades. Une prise de conscience du problème est en cours depuis une vingtaine d'année.

\section{H\&M : La France est-elle particulière en Europe sur cette histoire de recrutement des Africains dans son ancien Empire?}

Y. G. : Il faut revenir à l'histoire de la colonisation africaine où, effectivement, la France a une prépondérance sur le continent dès la fin du XIX ${ }^{\mathrm{e}}$ siècle. Sur le plan du football, l'originalité française fait que les liens des pays africains avec la France sont beaucoup plus forts qu'avec d'autres pays. Après la décolonisation, dans les années soixante et soixante-dix, la France était le seul pays à accueillir des footballeurs venus du continent africain, comme le montre le sommet Afrique-France organisé à Nice début juin 2010. À partir des années quatre-vingt, l'ouverture du marché des footballeurs fait que la France reste un réceptacle de nombreux footballeurs africains, mais aussi un espace de transit avant de partir jouer dans d'autres pays européens comme l'Angleterre, l'Espagne, l'Italie. Si par le passé la France a été le pré carré des footballeurs africains, aujourd'hui c'est l'Europe dans son ensemble qui reçoit ces footballeurs. 